\title{
Recipient hypertonic saline infusion prevents cardiac allograft dysfunction
}

\author{
Roberto V. P. Ribeiro, MD, ${ }^{a}$ Mitesh V. Badiwala, MD, PhD, ${ }^{a}$ Danny Ramzy, MD, PhD, ${ }^{b}$ \\ Laura C. Tumiati, BSc, ${ }^{a}$ and Vivek Rao, MD, $\mathrm{PhD}^{\mathrm{a}}$
}

\section{ABSTRACT}

Objective: Hypertonic saline (HTS) has potent immune and vascular effects. We assessed recipient pretreatment with HTS on allograft function in a porcine model of heart transplantation and hypothesized that HTS infusion would limit endothelial and left ventricular (LV) dysfunction following transplantation.

Methods: Heart transplants were performed after 6 hours of cold ischemic storage. Recipient pigs were randomized to treatment with or without HTS $(7.5 \% \mathrm{NaCl})$ before cardiopulmonary bypass $(\mathrm{CPB})$. Using a myograft apparatus, coronary artery endothelial-dependent (Edep) and -independent (Eind) relaxation was assessed. LV performance was determined using pressure-volume loop analysis. Pulmonary interleukin (IL)-2, IL-6, and tumor necrosis factor (TNF)- $\alpha$ expression was measured.

Results: Weaning from $\mathrm{CPB}$ and $\mathrm{LV}$ performance after transplantation were improved in HTS-treated animals. Successful weaning from CPB was greater in the HTS-treated hearts ( 8 of 8 vs 2 of $8 ; P<.05$ ). Mean LV functional recovery was improved in the HTS-treated animals, as assessed by preload recruitable stroke work $(65 \pm 10 \%$ vs $27 \pm 10 \% ; P<.001)$ and end-systolic elastance $(55 \pm 7 \%$ vs $37 \pm 4 \% ; P<.001)$. Treatment with HTS resulted in improved Edep (mean maximum elastance [Emax], $56 \pm 5 \%$ vs $37 \pm 7 \% ; P<.001$ ) and Eind (mean Emax \%, $77 \pm 6 \%$ vs $52 \pm 4 \% ; P<.001$ ) vasorelaxation compared with control. Pulmonary expression of IL-2, IL-6, and TNF- $\alpha$ increased following transplantation, whereas HTS therapy attenuated IL production $(P<.001)$. Transplantation increased plasma TNF- $\alpha$ levels and LV TNF- $\alpha$ expression, whereas HTS prevented this up-regulation $(P<.001)$.

Conclusions: Recipient HTS pretreatment preserves allograft vasomotor and LV function, and HTS therapy limits CPB-induced injury. HTS may be a novel recipient intervention to prevent graft dysfunction. (J Thorac Cardiovasc Surg $2019 ; 157: 615-25)$

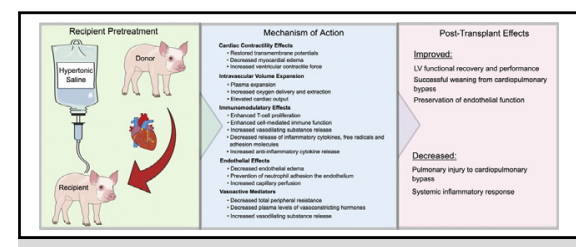

Beneficial effects of hypertonic saline infusion in cardiac transplant recipients.

\section{Central Message}

Hypertonic saline infusion may improve myocardial and endothelial function, as well as reduce inflammatory response. This may represent a simple method to improve clinical outcomes following cardiac transplantation.

\section{Perspective}

Ischemic injury to the myocardium sustained during organ procurement and storage has been related to development of primary graft failure and progression of graft coronary disease. Recipient hypertonic saline infusion can improve myocardial and endothelial function as well as reduce the recipient's inflammatory response, providing a simple and efficacious method to improve outcomes following cardiac transplantation.

See Editorial page 626.

See Editorial Commentary page 628.
Cardiac transplantation remains the gold standard treatment for eligible patients with end- stage heart disease. ${ }^{1}$ In clinical practice, cardiac allografts are arrested with a crystalloid cardioplegic solution and stored on ice after removal from the donor. Ischemic times of up to 4 hours are generally well tolerated; however, ischemic times $>6$ hours have been associated with poorer outcomes. ${ }^{2}$

\footnotetext{
From the ${ }^{a}$ Heart Transplant Program, Peter Munk Cardiac Centre, Toronto General Hospital, University Health Network, Division of Cardiovascular Surgery, University of Toronto, Toronto, Ontario, Canada; and ${ }^{\mathrm{b}}$ Division of Cardiothoracic Surgery, Cedars-Sinai Heart Institute, Los Angeles, Calif.

Supported by the Heart and Stroke Foundation of Ontario (Grant NA 5868), the Thoracic Surgery Foundation for Research and Education, the Tailored Advanced Collaborative Training in Cardiovascular Science for Research Fellows. DR was a Research Fellow of the TSFRE and TACTICS.
}

Unfortunately, primary graft dysfunction remains the predominant cause of early morbidity and mortality following cardiac transplantation., ${ }^{2,3}$ The causes of primary graft dysfunction are multifactorial and include the use of marginal organs, ischemia-reperfusion (I/R) injury sustained at the time of transplantation, recipient pulmonary vascular disease, and hyperacute rejection. ${ }^{3}$ In

Received for publication Nov 2, 2017; revisions received June 8, 2018; accepted for publication July 6, 2018; available ahead of print Aug 16, 2018.

Address for reprints: Vivek Rao, MD, PhD, 4MB-457, Toronto General Hospital, 200 Elizabeth St, Toronto M5G 2C4, Ontario, Canada (E-mail: vivek.rao@uhn.ca). $0022-5223 / \$ 36.00$

Copyright $@ 2018$ by The American Association for Thoracic Surgery https://doi.org/10.1016/j.jtcvs.2018.07.018 

Abbreviations and Acronyms
$\mathrm{CAV}=$ cardiac allograft vasculopathy
Cmax = maximum contraction
$\mathrm{CPB}=$ cardiopulmonary bypass
$\mathrm{CVP}=$ central venous pressure
Ees = end-systolic elastance
Emax $=$ maximum elastance
eNOS $=$ nitric oxide synthase
ET-1 = endothelin-1
HTS = hypertonic saline
IL = interleukin
$\mathrm{I} / \mathrm{R}=$ ischemia-reperfusion
LAD $=$ left anterior descending artery
$\mathrm{LV}=$ left ventricular
MPO = myeloperoxidase
$\mathrm{NaCl}=$ sodium chloride
PRSW $=$ preload-recruitable stroke work
SNP = sodium nitroprusside
TGF $=$ transforming growth factor
$\mathrm{TNF}=$ tumor necrosis factor

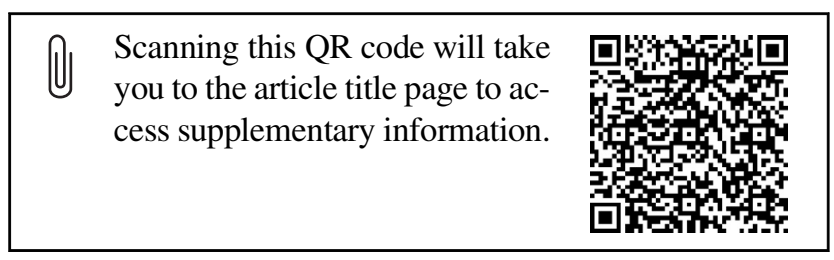

addition to early morbidity, cardiac transplant recipients face late morbidity primarily from lymphoproliferative disease, infection, and cardiac allograft vasculopathy (CAV). CAV occurs in approximately $50 \%$ of transplant recipients within 3 to 4 years, and evidence suggests that ischemic injury to the endothelium sustained at the time of organ procurement and storage is related to the development and progression of $\mathrm{CAV}^{4,5}$

Several investigators have evaluated novel methods of organ preservation with the aim of reducing the incidence of primary graft dysfunction and the development of late $\mathrm{CAV}^{6-9}$ Most previous studies have focused on new techniques and/or additives aimed specifically at donor organ preservation. Few investigations have examined the recipient as a potential target for therapeutic intervention. We previously developed a novel donor myocardial protective strategy that results in improved myocardial and endothelial function compared with standard storage techniques $^{6-8}$; however, this method is specific to the donor organ and does not affect the recipient immune system.

Hypertonic saline (HTS) has been shown to have immunomodulatory effects. ${ }^{10,11}$ Furthermore, several studies have demonstrated that HTS can reduce I/R injury. ${ }^{12-14}$ We have previously demonstrated in a porcine model that HTS administered to the donor before organ harvest improved cardiac functional recovery following transplantation. ${ }^{15} \mathrm{We}$ believe that potential exists for the clinical application of HTS to the recipient as a simple method of myocardial protection and immunomodulation. In this study, we examined the effects of recipient treatment with HTS on functional and immunologic responses following orthotopic heart transplantation in a preclinical porcine model.

\section{METHODS}

All experimental protocols were in accordance with federal and provincial regulations, policies, and guidelines. Female Yorkshire pigs $(\sim 60 \mathrm{~kg})$ were used to perform 16 orthotopic cardiac transplantations. All allograft donor hearts were stored under static hypothermic $\left(4^{\circ} \mathrm{C}\right)$ storage conditions in crystalloid cardioplegic solution for 6 hours. At 1 hour before recipient aortic crossclamping, animals were randomized to receive either an intravenous infusion of $4.5 \mathrm{~mL} / \mathrm{kg}$ of $7.5 \% \mathrm{NaCl}$ at $25 \mathrm{~mL} /$ minute (recipient HTS group; $\mathrm{n}=8$ ) or $4.5 \mathrm{~mL} / \mathrm{kg}$ of $0.9 \% \mathrm{NaCl}$ at $25 \mathrm{~mL} /$ minute (control group; $\mathrm{n}=8$ ).

\section{Donor Procedure}

Donor and recipient procedures were performed as described previously by our group. ${ }^{15}$ In brief, the animals were anesthetized with intramuscular ketamine $(30 \mathrm{mg} / \mathrm{kg})$ and inhalational isofluorane (1\%-5\%). Following median sternotomy, umbilical tapes were placed around the superior and inferior vena cava to permit adjustment of cardiac preload by caval snaring. A small apical ventriculotomy was created to permit insertion of an intraventricular Millar pressure catheter and a conductance catheter for continuous measurements of the left ventricular (LV) pressure-volume relationship. Pulmonary vascular resistance was assessed using a Swan-Ganz catheter. Baseline hemodynamic and contractility measurements were obtained.

After crossclamping, $1 \mathrm{~L}$ of Celsior solution was infused into the aortic root at $4{ }^{\circ} \mathrm{C}$ to achieve cardioplegic arrest, closely mimicking our current clinical practice. Following arrest, the donor heart was extracted, placed in a jar containing $1 \mathrm{~L}$ of hypothermic cardioplegic solution, and stored on ice.

\section{Recipient Procedure}

Initiation of the recipient operation was adjusted according to the time of donor organ procurement for a cold ischemic time of 6 hours, as described previously. ${ }^{15}$ Previous studies from our laboratory have demonstrated that $<20 \%$ of hearts will successfully maintain adequate hemodynamics following even shorter periods of ischemia. ${ }^{6-8}$ At 1 hour before recipient crossclamping and cardiectomy, HTS or $0.9 \% \mathrm{NaCl}$ was infused as described above. After the recipient aorta was crossclamped, the donor heart was removed from storage, and an initial blood cardioplegic dose of $350 \mathrm{~mL}$ was infused into the donor aortic root at a flow rate of $100 \mathrm{~mL} /$ minute. Cardioplegic protection consisting of a 2:1 mixture of blood/crystalloid was delivered at $10^{\circ} \mathrm{C}$ after the completion of each atrial anastomosis. After the completion of pulmonary arterial anastomosis, another $350-\mathrm{mL}$ blood cardioplegic dose was delivered at $37^{\circ} \mathrm{C}$. Orthotopic transplantations using a standard atria-to-atria technique were performed.

Once all anastomoses were completed, the aortic crossclamp was removed, and the heart was reperfused for 60 minutes. After reperfusion, $1 \mathrm{~g}$ of calcium chloride was given to all animals, and weaning from CPB was attempted. Isoproterenol infusion ( $4 \mathrm{~g} /$ minute) was provided as necessary to aid weaning from CPB. Weaning was deemed successful if the animal maintained a mean systemic pressure of $60 \mathrm{mmHg}$ for 30 minutes.

Immediately after complete weaning from CPB (and before the administration of any vasoactive agents), hemodynamic parameters were recorded and LV function was assessed as described below. After 
determination of success/failure to wean from CPB the hearts were excised under general anesthesia for assessment of coronary endothelial function.

\section{Outcome Analysis}

Ventricular functional assessment. A micromanometer and conductance catheter (Millar Instruments, Houston, Tex) were used for construction of pressure-volume relationships using the methods of Baan and colleagues, ${ }^{16}$ and parallel conductance was corrected by the method of Szwarc and colleagues. ${ }^{17}$ Measurements were then taken at baseline in the donor animal and immediately after discontinuation of CPB. End-systolic elastance (Ees) was calculated as the slope of the line of the end-systolic points of the pressure-volume loops. Preload-recruitable stroke work (PRSW) was evaluated by calculating the relationship between stroke work and end-diastolic volume using IOX version 1.8.9.13 (EMKA Technologies, Falls Church, Va).

Endothelial functional assessment. Endothelial-dependent and -independent vascular relaxation were assessed in vitro by constructing concentration-response curves with a small-vessel myograph for isometric tension recording using left anterior descending artery rings as described previously (Supplementary Materials). ${ }^{15}$ Control assessments were performed on coronary segments obtained from the recipient's native heart (normal group).

Biochemical analysis. Myocardial metabolism was assessed by obtaining blood samples and full-thickness LV myocardial biopsy specimens from the ventriculotomy. Simultaneous arterial, mixed venous, and coronary sinus blood samples were obtained immediately before recipient crossclamping (baseline) and at 5 and 60 minutes of reperfusion (after opening of the recipient crossclamp). In addition, blood samples were obtained every 5 minutes for 1 hour during HTS infusion before recipient crossclamping.

Myocardial biopsy specimens obtained at baseline and at the end of the experiment were assessed for markers of oxidative stress (8-isoprostane and myeloperoxidase [MPO] activity), and endothelial injury (TNF- $\alpha$, endothelin-1 [ET-1], and nitric oxide synthase [eNOS] activity). Lung biopsy specimens were obtained from the recipient animals before HTS infusion (baseline control) and at 5 and 60 minutes after transplantation. Lung tissue TNF- $\alpha$, IL-2, IL-6, and IL-10 were detected by standard Western blot techniques. Full details are provided in the Supplementary Materials.

\section{Statistical Analysis}

Statistical analysis was performed using SAS version 9.0 (SAS Institute, Cary, NC). Categorical data were analyzed using the 2-tailed Fisher exact test where appropriate. Continuous data are expressed as mean \pm standard deviation and were analyzed by 2 -way repeated-measures analysis of variance, evaluating the main effects of group and time as well as the interactive effect (group-time).

\section{RESULTS}

\section{Operative Parameters}

There were no between-group differences in storage time or total ischemic time, because these was controlled by the experimental design (data not shown). Similarly, there were no significant between-group differences in CPB time or recipient crossclamp time (data not shown).

\section{Effects of HTS Infusion on Sodium Concentration}

Figure 1, $A$, illustrates the serum sodium concentration at baseline and during the hour after completion of HTS or normal saline infusion. Administration of HTS increased the mean serum sodium level from $139 \pm 2 \mathrm{mmol} / \mathrm{L}$ to
$155 \pm 3 \mathrm{mmol} / \mathrm{L}(P<.001)$, which normalized to $144 \pm 3 \mathrm{mmol} / \mathrm{L}$ by 1 hour postinfusion (Figure 1, $A$ ).

\section{Hemodynamic Effects of HTS Infusion Versus Normal Saline Infusion}

No differences were noted in systemic pressures after HTS administration compared with controls $(P=.08$; Figure $1, B)$. HTS infusion resulted in a transient increase in pulmonary pressure compared with the normal saline-infused group $(P<.05$; Figure $1, C)$. In addition, HTS was associated with a significant increase in the cardiac index compared with control $(P<.01$; Figure 1 , $D)$. These hemodynamic changes returned to baseline, with no differences between groups by 1 hour postinfusion. Therefore, before recipient crossclamping, all parameters were similar in the 2 groups.

\section{Biochemical Parameters}

Lactate levels. Myocardial lactate production was not significantly different between groups at baseline before donor cardioplegic arrest (mean, $1.3 \pm 0.3 \mathrm{mmol} / \mathrm{L}$ for control vs $1.34 \pm 0.3 \mathrm{mmol} / \mathrm{L}$ for HTS; Figure $2, A$ ). Following transplantation, a significant increase was observed in myocardial lactate release after 5 minutes, with a further rise after 60 minutes of reperfusion (Figure 2, A). HTS treatment significantly limited lactate production compared with the control group at 60 minutes of reperfusion (mean, $7.0 \pm 0.2 \mathrm{mmol} / \mathrm{L}$ for control vs $5.0 \pm 0.2 \mathrm{mmol} / \mathrm{L}$ for HTS; $P<.001$; Figure $2, A$ ).

Mixed venous lactate level showed no significant between-group differences at prearrest. In both groups, a significant rise in mixed venous lactate levels was observed after 5 and 60 minutes of reperfusion $(P<.001$; Figure $2, A)$. Total body lactate release was also reduced by HTS treatment at 60 minutes of reperfusion compared with control (mean, $7.0 \pm 0.4 \mathrm{mmol} / \mathrm{L}$ for control vs $5.5 \pm 0.3 \mathrm{mmol} / \mathrm{L}$ for HTS; $P<.001$; Figure $2, A$ ).

\section{Oxidative Injury}

Pulmonary oxidative injury was assessed by measuring MPO levels in lung biopsy specimens as a percent increase from baseline (Figure 2, B). MPO levels increased significantly after 5 and 60 minutes of cardiac allograft reperfusion in the control recipients. HTS therapy limited the CPB-induced rise in pulmonary MPO levels ( 5 minutes: mean, $144 \pm 6 \%$ in control vs $88 \pm 4 \%$ in HTS; 60 minutes: mean, $106 \pm 8 \%$ in control vs $86 \pm 2 \%$ in HTS; $P<.001$ for both).

Cardiac oxidative stress was also assessed in myocardial samples by measuring 8-isoprostane levels as a percent increase from baseline (Figure 2,C). The 8isoprostane levels were assessed immediately after cardioplegic arrest and again after 60 minutes of reperfusion following transplantation. Levels were increased 

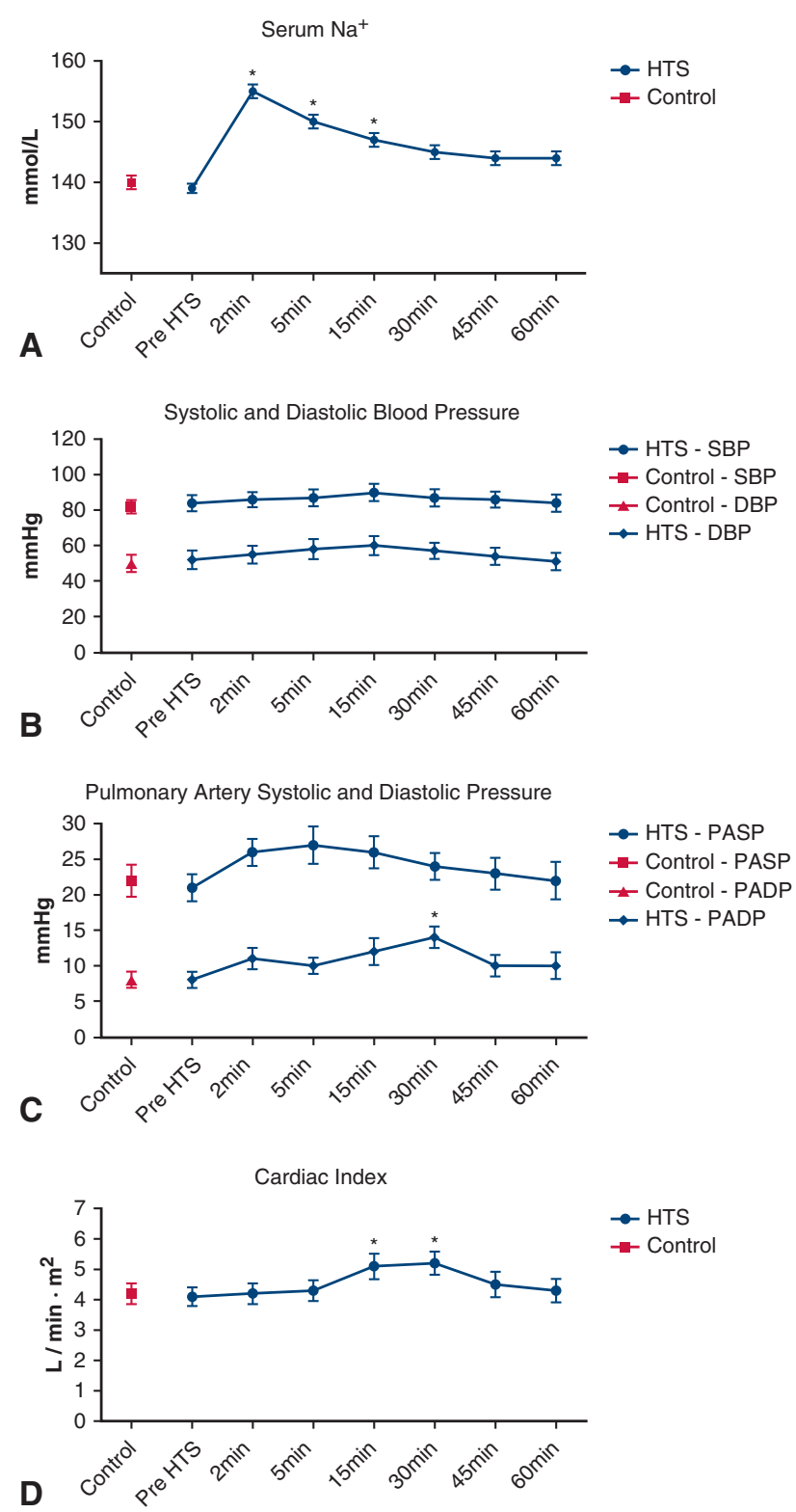

FIGURE 1. Effects of hypertonic saline (HTS) infusion. A, Administration of hypertonic saline acutely increased serum sodium level, with subsequent normalization by 1 hour postinfusion $(P<.01)$. B, Systemic pressure. HTS resulted in no changes in systemic pressure compared with controls $(P=.08)$. C, Pulmonary pressure. HTS resulted in a transient rise in pulmonary pressures, with normalization by 1 hour postinfusion. $* P<.05 . \mathrm{D}$, Cardiac index. HTS increased cardiac index transiently, with normalization within 1 hour postinfusion. $* P<.01$. $S B P$, Systolic blood pressure; $D B P$, diastolic blood pressure; $P A S P$, pulmonary artery systolic pressure; $P A D P$, pulmonary artery diastolic pressure.

significantly after reperfusion in both groups. HTS treatment significantly limited the rise compared with control at 60 minutes (mean, $91 \pm 9 \%$ in control vs $61 \pm 6 \%$ in HTS; $P<.001)$.

\section{Inflammatory Markers}

Coronary sinus blood levels of TNF- $\alpha$ were compared between our groups at prearrest and at 5 and 60 minutes after reperfusion (Figure 3). No significant between-group difference was observed before cardioplegic arrest (mean, $40 \pm 2 \mathrm{pg} / \mathrm{mL}$ vs $36 \pm 6 \mathrm{mmol} / \mathrm{L} ; P=.096$ ). Cardiac transplantation significantly increased TNF- $\alpha$ levels following 5 and 60 minutes of reperfusion in both groups (time effect, $P<.01)$. HTS therapy limited the release of TNF- $\alpha$ compared with control at 5 and 60 minutes of reperfusion (group effect, $P<.01$; Figure 3 ).

ET-1 levels in coronary sinus blood rose significantly after 5 minutes of reperfusion, and these levels remained high after 60 minutes (Figure 3). HTS treatment significantly reduced ET-1 levels by a mean of $25 \pm 9 \%$ after 5 minutes and by $17 \pm 9 \%$ after 60 minutes compared with control $(P<.01)$.

Pulmonary IL-2, IL-6, IL-10, and TNF- $\alpha$ protein levels were significantly increased after reperfusion (Figure 4, A). IL-2, IL-6, and IL-10 were up-regulated significantly from baseline after 5 minutes of reperfusion $(P<.001)$. After 60 minutes, all IL levels decreased; however, protein expression remained significantly higher than baseline $(P<.001)$. HTS-exposed recipients demonstrated significantly reduced up-regulation of IL-2, IL-6, and IL-10 expression in pulmonary tissue compared with control $(P<.001)$. Pulmonary tissue TNF- $\alpha$ expression was increased after transplantation, with HTS therapy significantly reducing expression $(P<.001$; Figure $4, A)$.

Myocardial expression of TNF- $\alpha$ and transforming growth factor (TGF) $\beta$ were significantly up-regulated after 60 minutes of reperfusion (TNF- $\alpha, 32 \pm 3 \%$; TGF- $\beta$, $25 \pm 4 \% ; \quad P<.001)$. HTS therapy attenuated transplantation-induced TNF- $\alpha$ up-regulation, whereas TGF- $\beta$ expression following reperfusion was downregulated by $14 \pm 3 \%(P<.001$; Figure $4, B)$.

\section{Myocardial NOS Expression}

In the control group, myocardial expression of eNOS was down-regulated by $13 \pm 4 \%$ after transplantation, whereas HTS-exposed animals showed a significant up-regulation of eNOS expression $(40 \pm 3 \%)(P<.001$; Figure $4, B)$. No significant between-group differences in inducible NOS expression were observed (Figure 4, B).

\section{Function}

The rate of successful weaning from CPB was greater in the HTS-treated animals compared with controls ( 8 of 8 vs 2 of $8 ; P<.003$ ). The use of inotropic support following transplantation was reduced with HTS treatment ( 2 of 8 vs 8 of $8 ; P<.003$ ). As shown in Figure 5, LV functional recovery was improved in the HTS-treated animals after transplantation, as assessed by PRSW, Ees, 


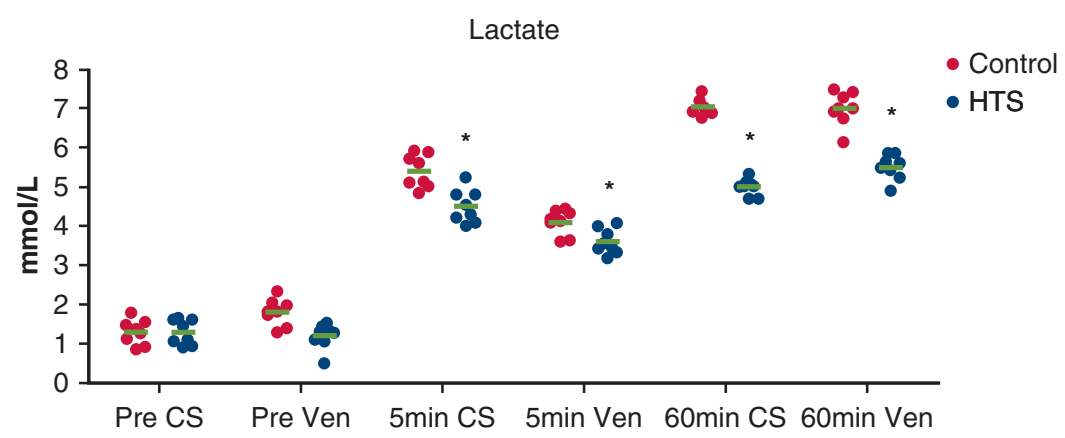

A

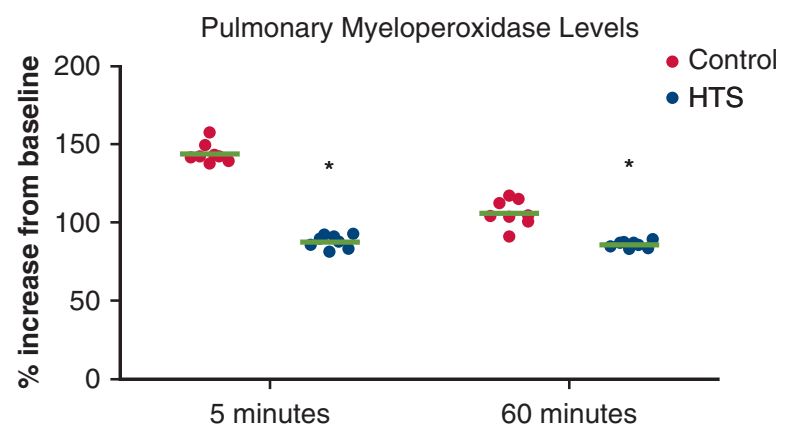

B

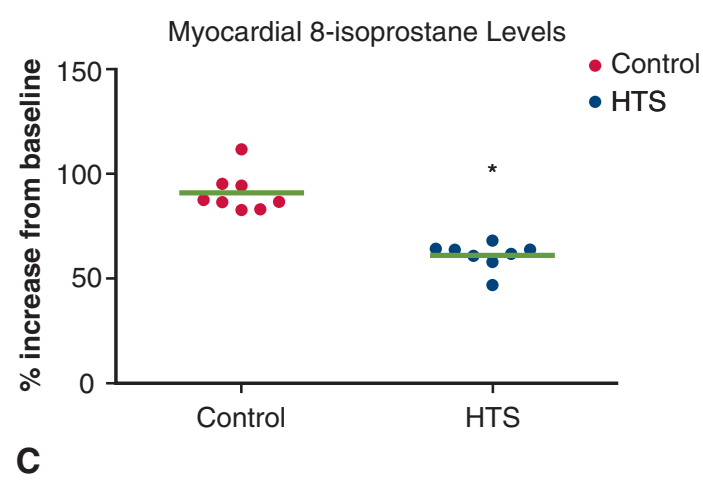

FIGURE 2. A, Lactate levels. Mixed venous (ven) and coronary sinus (CS) lactate production increased following transplantation. Hypertonic saline (HTS) treatment attenuated lactate production. $* P<.001$ versus control. B, Pulmonary tissue myeloperoxidase (MPO) levels. MPO levels were increased posttransplantation, with HTS treatment limiting MPO production. $* P<.01$ versus control. C, Myocardial 8-iosprostane levels. HTS treatment limited oxidative injury compared with control. $* P<.01$ versus control.

and maximum elastance (Emax). HTS treatment increased PRSW posttransplantation recovery compared with control animals successfully weaned off CPB (mean \%recovery, $65 \pm 10 \%$ for HTS vs $5 \pm 12 \%$ for control vs $27 \pm 10 \%$ for weaned control; $P<.001)$. Similarly, recovery of Ees was significantly higher in the HTS group compared with control and weaned control animals (mean \%recovery, $55 \pm 7 \%$ for HTS vs $7 \pm 16 \%$ for control vs $37 \pm 4 \%$ for weaned control; $P<.001$ ). The load-sensitive index, Emax \%, was also significantly different between groups; HTS better preserved Emax compared with both control and weaned control animals $(P<.001)$.

\section{Hemodynamics}

Figure 6 shows hemodynamic values measured in the recipient animals pretransplantation and postreperfusion. HTS exposure prevented the rise in central venous pressure and pulmonary capillary wedge pressure seen in the control group following reperfusion. In addition, HTS treatment significantly increased the posttransplantation CI compared with control $(P<.001)$. Improved systemic pressure and lower pulmonary pressures were observed following transplantation in the HTS animals compared with control $(P<.001)$.

\section{Coronary Vasomotor Function}

Figure 7 shows the dose-response curves of left anterior descending artery (LAD) segments to bradykinin, sodium nitroprusside, and ET-1 as measures of endothelial-dependent vasorelaxation, endothelial-independent vasorelaxation, and vasospasm, respectively. Allograft storage and transplantation resulted in impaired endothelial-dependent vasodilation (mean Emax \%, $56 \pm 5 \%$ for normal vs $37 \pm 7 \%$ for control; $P<.001$; Figure 7, A). HTS treatment better prevented endothelial-dependent vasomotor dysfunction compared with control (mean Emax\%, $60 \pm 6 \%$ for HTS vs $37 \pm 7 \%$ for control; $P<.001$; Figure $7, A$ ). HTS therapy also better abrogated endothelial-independent dysfunction compared with control (mean Emax \%, $77 \pm 6 \%$ for HTS vs $52 \pm 4 \%$ for control; $P<.001$; Figure $7, B)$. HTS-treated animals had similar endothelial-dependent and independent vasomotor function as normal nontransplanted animals. Sensitivity to ET-1-induced vasospasm was increased following transplantation (mean maximum contraction [Cmax\%], $221 \pm 19 \%$ in normal vs $530 \pm 47 \%$ in control; $P<.001$; Figure $7, C)$. HTS treatment reduced vasospasm response compared with control (mean Cmax \%, $340 \pm 28 \%$ for HTS vs $530 \pm 47 \%$ for control; $P<.001$; Figure $7, C)$. 


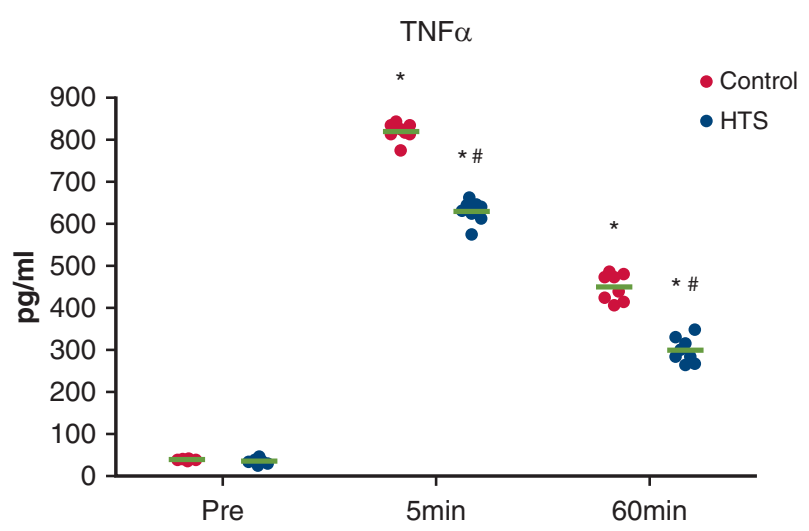

A

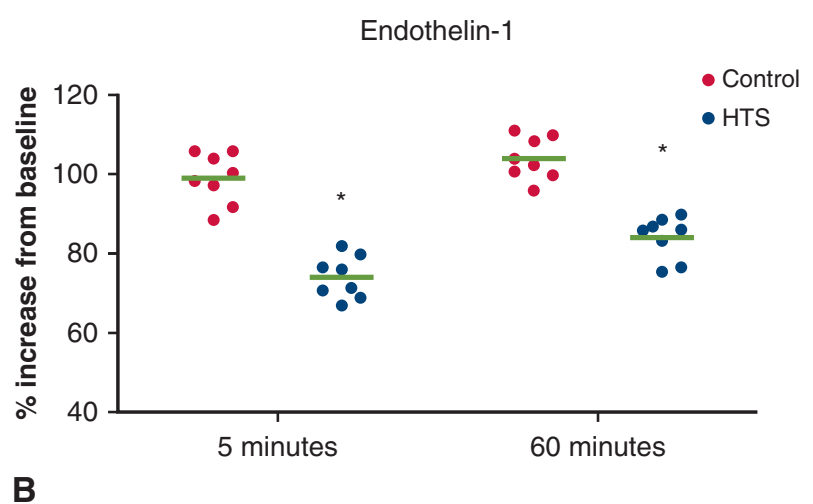

FIGURE 3. A, Coronary sinus blood plasma tumor necrosis factor $(T N F)-\alpha$ levels. Plasma TNF- $\alpha$ levels in coronary sinus effluent after 5 minutes and 60 minutes of reperfusion were significantly reduced with recipient hypertonic saline $(H T S)$ treatment. $* P<.05$ versus pre-reperfusion; ${ }^{\#} P<.05$ versus control. B, Plasma endothelin-1 (ET-1) levels. ET-1 levels increased posttransplantation, with HTS therapy attenuating ET-1 release. $* P<.001$ versus control.

\section{DISCUSSION}

Ischemic injury to both myocardium and endothelium sustained at the time of organ procurement and storage had been related to the development of primary graft failure and the progression of graft coronary disease. ${ }^{2-5,18,19} \mathrm{I} / \mathrm{R}$ injury during organ retrieval and transplantation has several direct deleterious effects, including activation of both leukocytes and endothelial cells, resulting in liberation of reactive oxygen species and a wide variety of inflammatory cytokines. ${ }^{4,5,20-22}$ Endothelial activation from a wide variety of stimuli also may lead to the development of CAV. ${ }^{4,18}$ Therefore, improved methods of myocardial protection specifically aimed at endothelial preservation and inhibition of cytokine release may reduce the incidence of primary graft dysfunction and CAV. To our knowledge, we are the first group to demonstrate the beneficial effects of hypertonic saline infusion in cardiac transplant recipients, which we have summarized in the Central Picture.

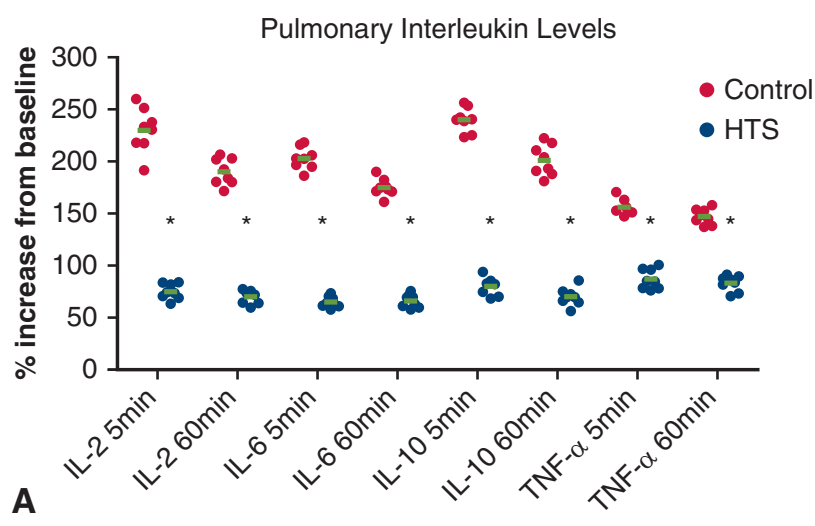

Myocardial Inflammatory Cytokines

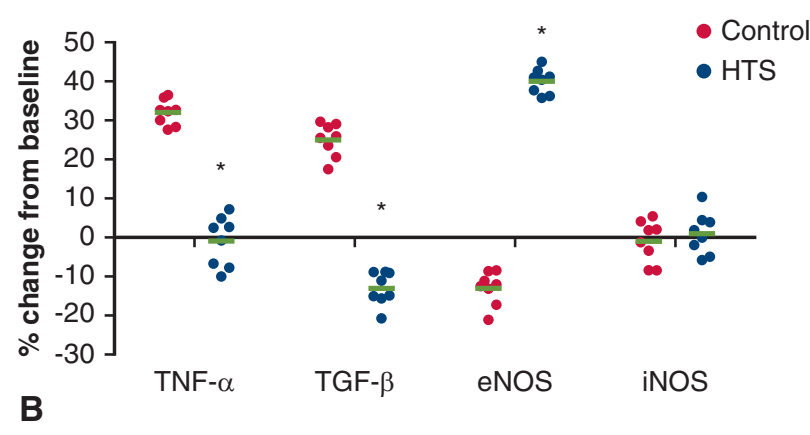

FIGURE 4. A, Pulmonary tissue interleukin (IL) levels. Hypertonic saline (HTS) treatment limited the production of IL-2, IL-6, IL-10, and tumor necrosis factor $(T N F)-\alpha$ compared with control after both 5 minutes and 60 minutes of reperfusion. $* P<.001$ versus control. B, Myocardial expression of inflammatory cytokines. HTS treatment prevented posttransplantation TNF- $\alpha$ and transforming growth factor (TGF)- $\beta$ up-regulation. ${ }^{*} P<.001$ versus control. Nitric oxide synthase $(e N O S)$ expression was up-regulated posttransplantation compared with control myocardia, which demonstrated eNOS down-regulation. No significant differences were observed in inducible NOS (iNOS) expression.

Allograft contractile performance was evaluated using load-insensitive measures of LV systolic function. HTS treatment enhanced LV functional recovery after transplantation, as assessed by both PRSW and Ees. Importantly, the PRSW relationship is a sensitive measure of porcine allograft LV functional recovery after orthotopic transplantation. Transplanted hearts revealed a characteristic downward and rightward shift after transplantation, indicating LV contractile dysfunction. These findings are similar to those from previous studies published by our group. ${ }^{6,7,15}$ Porcine hearts are extremely sensitive to ischemia, and standard organ preservation techniques fail to consistently preserve the porcine heart even after 4 hours of hypothermic storage. ${ }^{8}$ However, the decrease in PRSW was attenuated in the HTS-treated animals, indicating beneficial effects on LV functional recovery. This improvement was associated with a 4-fold increase in the ability to wean from CPB and a simultaneous 


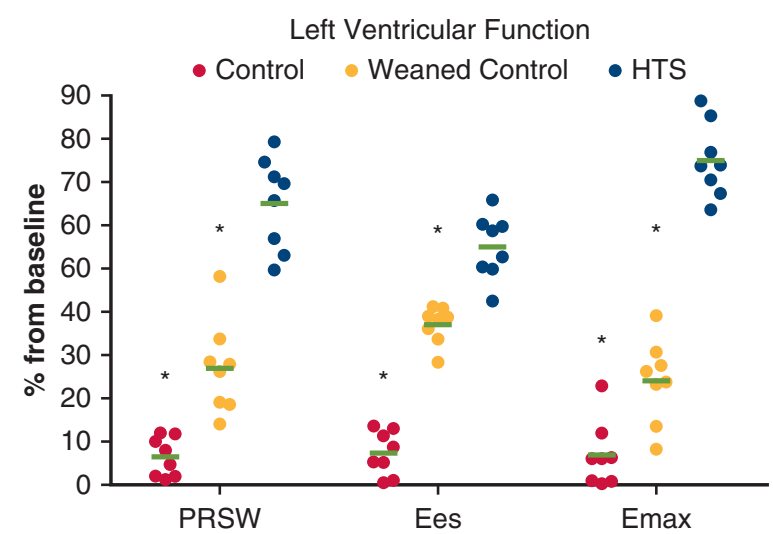

FIGURE 5. Left ventricular (LV) functional assessment. Hypertonic saline (HTS) treatment enhanced LV functional recovery after orthotopic transplantation. Two independent measures and a load-insensitive measure of LV performance indicated improved functional recovery in the HTS group. Preload recruitable stroke work $(P R S W)$ posttransplantation was improved compared with control. $* P<.001$. Similarly, end-systolic elastance (Ees) was improved compared with control. $* P<.001$. HTS-exposed animals demonstrated improved recovery compared with weaned controls. $* P<.001$. Emax, Maximum elastance.

4-fold decrease in the need for inotropic support. The ability to wean these hearts after 6 hours of static hypothermic storage with less support further emphasizes the efficacy of this treatment. When we analyzed the control animals that had been successfully weaned from CPB, these hearts also displayed significantly poorer LV functional recovery compared with HTS-treated animals. Therefore, HTS increased our ability to wean from $\mathrm{CPB}$ while simultaneously improving LV performance after cardiac transplantation.

Various groups have previously demonstrated the ability of HTS treatment to improve myocardial performance following I/R injury. Waagstein and colleagues ${ }^{14}$ found that HTS infusion improved myocardial performance during reperfusion. Sidi and colleagues ${ }^{23}$ demonstrated $^{2}$ the same protective effects against LV dysfunction in a porcine LAD occlusion model of myocardial ischemia and reperfusion. In a porcine model of resuscitation following cardiac arrest, Krep and colleagues ${ }^{24}$ demonstrated that HTS infusion enhanced both myocardial perfusion and recovery of function. Of more relevance to transplant recipients, we observed that HTS therapy lowered central venous pressure and pulmonary pressures while improving CI posttransplantation. Because right heart failure is a common problem after heart transplantation, ${ }^{3}$ it was particularly encouraging to observe these beneficial effects on pulmonary vascular resistance. The effects of HTS were not a result of improved preoperative hemodynamics, given that CPB hemodynamic parameters were similar between groups before initiation of CPB. Therefore, HTS treatment to the transplant recipient decreased myocardial I/R injury, providing improved LV functional recovery and hemodynamics after transplantation.

HTS also reduced anaerobic metabolism posttransplantation, as evidenced by decreased lactate release compared with control. Furthermore, HTS limited free radical injury, as demonstrated by decreased myocardial 8-isoprostane levels compared with control. Decreased oxidative stress and improved metabolism are potential mechanism by which HTS can limit myocardial dysfunction. eNOS up-regulation was noted in the HTS group. This up-regulation may result in improved LV performance by enhancing diastolic compliance and increasing coronary blood flow. Thus, HTS improved myocardial metabolism and decreased oxidative stress while up-regulating eNOS expression, leading to enhanced myocardial recovery posttransplantation.

Another potential mechanism in the early positive hemodynamic effects of HTS is via prevention of intracellular $\mathrm{Ca}^{2+}$ overload during initial reperfusion, as described by Deutsch and Kaczmarek. ${ }^{25}$ Harada and colleagues ${ }^{26}$ reported postischemic cardiac contractile dysfunction induced by intracellular $\mathrm{Ca}^{2+}$ accumulation. In their study, extracellular hypernatremia during early reperfusion led to improved contractility recovery, likely by preventing intracellular $\mathrm{Ca}^{2+}$ increase. These findings suggest the importance of cellular ion-exchange processes during initial organ reperfusion. ${ }^{25,27}$

\section{Endothelial Protection}

It is conceivable that improved vasomotor function could result in enhanced contractile function by improving myocardial perfusion and washout of toxic metabolites that accumulate during storage. Activated endothelial cells produce and release cytokines, which promote the adhesion of circulating leukocytes. During reperfusion, circulating leukocytes bind to the activated endothelial cells, migrate through the endothelial layer into the media, and start to produce oxygen-derived free radicals and toxic cytokines, such as TNF- $\alpha$ and TGF- $\beta$.

We observed increases in the allograft production of cytokines such as TNF- $\alpha$ and TGF- $\beta$ posttransplantation. We demonstrated significant attenuation of coronary sinus blood TNF- $\alpha$ and TGF- $\beta$ levels at the end of allograft reperfusion following HTS treatment, suggesting that HTS treatment may protect against endothelial cell activation within the allograft. We believe that if this endothelial activation can be attenuated, it may be possible to obviate much of the myocardial dysfunction seen early after cardiac transplantation. Zakaria and colleagues ${ }^{28}$ demonstrated that the endothelial protective effects of HTS in cellular studies translated into improved vascular function in a rat model of hemorrhagic shock. Thus, there is both in vitro and in vivo evidence that HTS protects against endothelial activation, resulting in improved endothelial function, which 

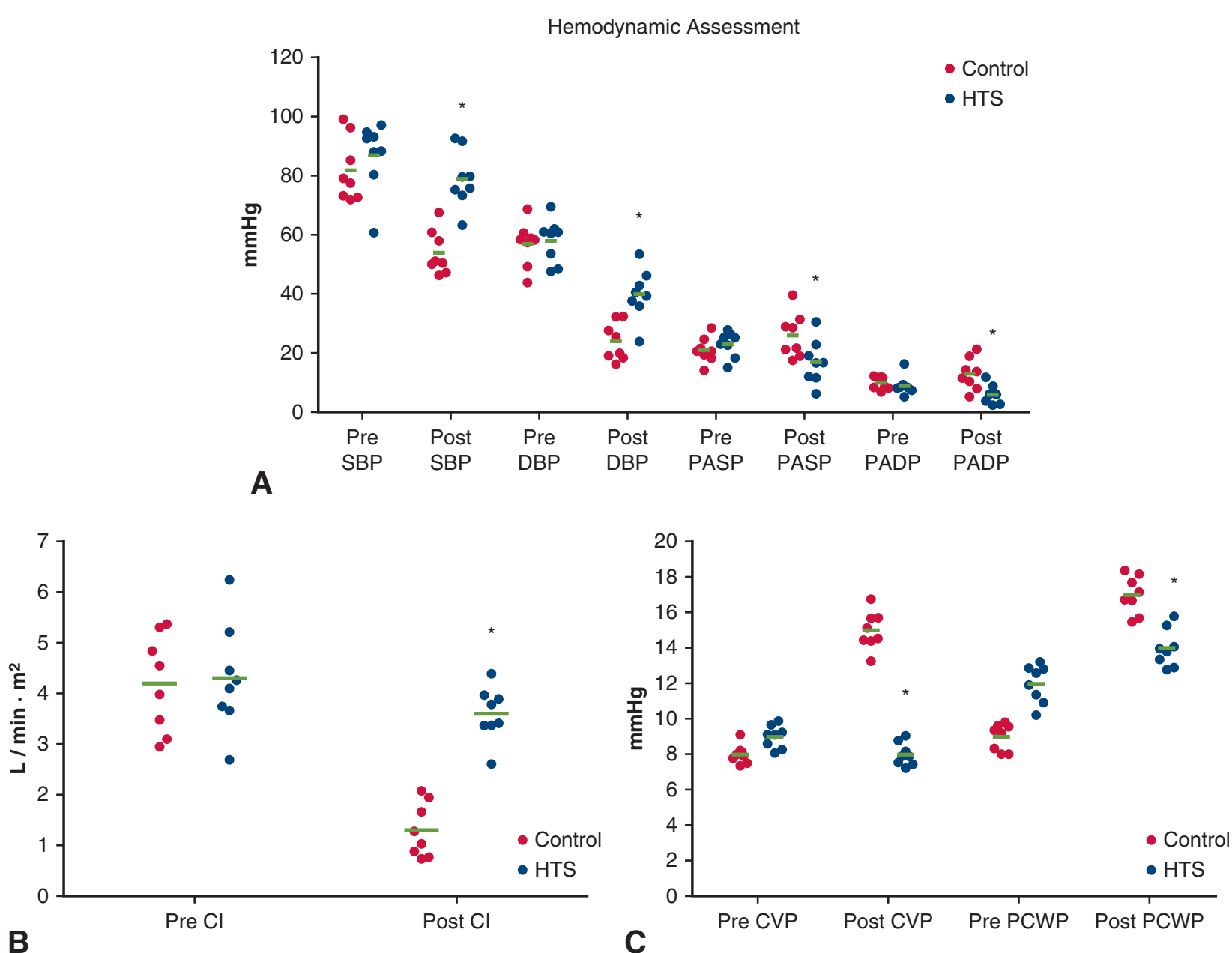

FIGURE 6. A, Systemic hemodynamic measurements pretransplantation and posttransplantation. Posttransplantation systemic blood pressure was significantly improved with hypertonic saline $(H T S)$ treatment. $* P<.001$ versus control. B, Pulmonary hemodynamic measurements pretransplantation and posttransplantation. Posttransplantation pulmonary blood pressure was significantly improved with HTS treatment. $* P<.001$ versus control. C, Cardiac index $(C I)$. HTS treatment significantly increased CI posttransplantation compared with controls. $* P<.001$ versus control. $S B P$, Systolic blood pressure; $D B P$, diastolic blood pressure; $P A S P$, pulmonary artery systolic pressure; $P A D P$, pulmonary artery diastolic pressure; $C V P$, central venous pressure; $P C W P$, pulmonary capillary wedge pressure.

may protect against vasomotor dysfunction in the setting of transplantation-related I/R injury.

In the present study, control LAD segments demonstrated significant impairment in endothelial-dependent and endothelial-independent vasorelaxation; HTS treatment preserved vasomotor function of these segments. We also assessed sensitivity to ET-1-induced vasospasm. Coronary artery sensitivity to vasospasm posttransplantation was significantly increased; however, HTS attenuated ET-1-induced vasospasm but failed to return vessel sensitivity to normal levels. The protective effects of HTS in limiting vasospasm may be greater in vivo, because in addition to reducing ET-1 sensitivity, HTS decreased ET-1 levels. These findings suggest that HTS treatment protects not only endothelial function, but also vascular smooth muscle cell functional integrity.
Improved vasomotor function posttransplantation exerts beneficial effects by increasing myocardial blood flow, oxygen delivery, and toxic metabolic washout, leading to improved myocardial performance. In addition, vasomotor protection early after transplantation may lead to decreased allograft vasculopathy. In a rodent model of orthotopic aortic allograft transplantation, Verrier and colleagues ${ }^{4}$ demonstrated that $\mathrm{I} / \mathrm{R}$ injury resulted in endothelial injury, leading to the development of transplant vasculopathy. They also found that endothelial cell loss occurred in both isografts and allografts due to $\mathrm{I} / \mathrm{R}$ injury. This initial loss of endothelial cells was replaced within 2 weeks; however, transplantation vasculopathy developed within 60 days. ${ }^{4}$ Their study indicated that I/R injury led to the development of transplantation vasculopathy, because isografts developed vasculopathy, although to a lesser extent than allografts. The 

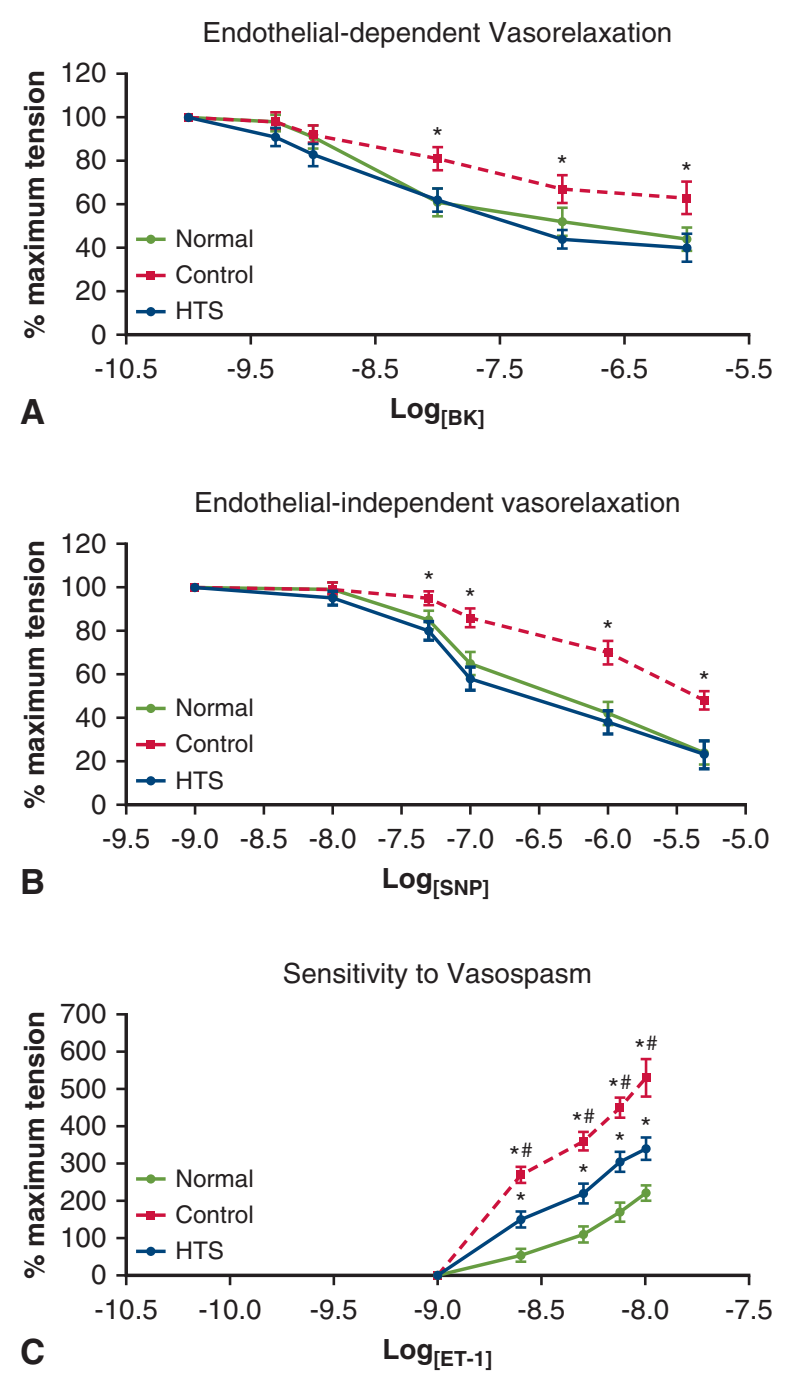

FIGURE 7. A, Endothelial-dependent vasodilation in porcine left anterior descending (LAD) coronary arteries. The graph depicts the cumulative dose-response curves for bradykinin (Bk) in LAD segments. Hypertonic saline (HTS) prevented endothelial-dependent vasomotor dysfunction compared with control. $* P<.001$ versus control. B, Endothelial-independent vasodilation of porcine LAD coronary arteries. The graph depicts the cumulative dose-response curves to sodium nitroprusside (SNP) in LAD segments. HTS prevented endothelial-independent vasomotor dysfunction compared with control. $* P<.001$ versus control. $C$, Sensitivity to vasospasm. Cumulative dose-response curves to endothelin-1 (ET-1) in LAD segments. HTS decreased vasosensitivity to ET-1 compared with control. $* P<.001$ versus normal; ${ }^{\#} P<.05$ versus HTS.

protective effect of HTS on vasomotor function and endothelial function may confer protection against the development of CAV in addition to myocardial preservation.

\section{HTS and ET-1}

Improved myocardial function can be achieved by modulating ET-1 homeostasis. We have previously demonstrated that elevated ET-1 level worsens I/R injury and results in impaired vascular function. ${ }^{29}$ Attenuating the release of ET-1 following I/R injury can enhance cardiomyocyte tolerance to I/R injury. ${ }^{29,30}$ Using a human cardiomyocyte cell model of simulated I/R injury, we have previously shown that ET-1 antagonism improves cell survival. ${ }^{29}$ Furthermore, ET-1 antagonism attenuates ischemic injury and improved vasomotor function. ${ }^{29}$ In a model similar to that used in the present study, we demonstrated that ET-1 antagonism improved LV function recovery. ${ }^{6}$ Therefore, the lower ET-1 levels seen with HTS therapy may be another mechanism by which improved LV function occurs in HTS-exposed animals.

\section{HTS and Inflammation}

The inflammatory response to cardiac transplantation has 3 crucial components. Initially, there is the inflammatory response as a result of $\mathrm{CPB}$, which leads to leukocyte activation, cytokine release, expression of adhesion molecules, and complement activation. ${ }^{31,32}$ The recipient response to CPB leads to native organ injury and, more importantly, aggravates allograft injury caused by procurement and storage. The second component is allograft reperfusion, resulting in immune system activation. Reperfusion injury is well documented and results in worsening of the injury occurring during ischemia as damaged endothelial cells become susceptible to the generation of reactive oxygen species. ${ }^{20,21,33,34}$ The third component is the well-recognized injury that occurs from allograft rejection. Allograft rejection occurs in all transplants to a varying degree. Limiting immune activation at any of these stages will improve both myocardial and endothelial protection. Techniques that beneficially modulate the immune response during cardiac transplantation may lead to improved clinical outcomes following surgery.

HTS exposure limited the deleterious effects of CPB. Total body lactate production was lower in the HTS group compared with the control group, indicating reduced total body ischemia and injury from CPB. Furthermore, HTS therapy decreased pulmonary oxidative injury, as shown by lower MPO levels in lung biopsy specimens. These data are further supported by the finding of lower 8 -isoprostane levels in myocardial tissue seen in the HTS-treated group.

HTS treatment also limited immune activation. Pulmonary IL-2, IL-6, IL-10, and TNF- $\alpha$ levels were significantly lower following HTS exposure compared with controls, demonstrating the ability of HTS therapy to modulate immune activation. In addition, coronary sinus plasma TNF- $\alpha$ levels were significantly reduced. Myocardial TNF- $\alpha$ and TGF- $\beta$ expression was down-regulated following HTS exposure. Therefore, HTS may be able to limit allograft dysfunction and possibly prevent or lessen the development of CAV by inhibiting immune/ inflammatory activation. 
HTS has the potential to limit both allograft- and CPB-induced injury. Thus, HTS therapy is not limited to the allograft, but may protect the entire patient from the deleterious effects of transplantation. In recent years, several investigators have also demonstrated in I/R models that HTS also exerts immunomodulatory effects. ${ }^{10-12,24}$ The immunomodulatory effects of HTS treatment may provide a novel strategy for myocardial and endothelial protection during cardiac allograft transplantation.

Our study has some important limitations worthy of note. First, as a preclinical large animal study, it had a relatively small sample size, and extrapolating our findings into a clinical setting should be done with caution, especially in transplant recipients with advanced heart failure. In additionally, some analyses might have been significant, such as the hemodynamic assessment during HTS infusion, with the inclusion of more animals. However, as demonstrated above, all changes during HTS infusion returned to baseline levels before recipient cardiectomy, and we do not believe this would have resulted in a different outcome with a larger sample size. The investigators were not blinded to the experimental groups; however, this did not influence outcomes, such as successful weaning from $\mathrm{CPB}$, because a strict protocol was followed to determine this. Finally, posttransplantation monitoring was limited to 30 minutes after discontinuation of CPB. Although our findings demonstrate an important effect of HTS over early posttransplantation cardiac performance and reperfusion injury, this cannot be extrapolated to an overall improved long-term outcome.

Our findings demonstrate the potential beneficial effects of HTS in both the recipient and the donor heart. A surprising finding from a previous study by our group evaluating direct donor allograft protection with HTS was that a recipientdirected intervention resulted in benefit to the allograft. ${ }^{15}$ The administration of HTS has been used safely in several clinical situations as well as in cardiac surgery patients. Previous studies not only demonstrated safety, but also showed potential beneficial effects, such as improved myocardial perfusion and hemodynamics. ${ }^{31,32}$ Our study indicates that HTS can be used to protect the recipient from CPBinduced injury and from allograft failure.

In summary, we believe that HTS infusions to the recipient will improve myocardial and endothelial function, as well as reduce the recipient's inflammatory response to cardiac transplantation. HTS treatment may represent a simple, cost-effective, and efficacious method to potentially improve early clinical outcomes following cardiac transplantation.

\section{Conflict of Interest Statement}

Dr Ramzy reports research scholarships from the Thoracic Surgery Foundation for Research and Education and Collaborative Training in Cardiovascular Science for
Research Fellow during the conduct of this study. All other authors have nothing to disclose with regard to commercial support.

\section{References}

1. Messer S, Ardehali A, Tsui S. Normothermic donor heart perfusion: current clinical experience and the future. Transpl Int. 2015;28:634-42.

2. Lund LH, Edwards LB, Kucheryavaya AY, Benden C, Christie JD, Dipchand AI, et al. The registry of the International Society for Heart and Lung Transplantation: thirty-first official adult heart transplant report, 2014; focus theme: retransplantation. J Heart Lung Transplant. 2014;33:996-1008.

3. Cosío Carmena MD, Gómez Bueno M, Almenar L, Delgado JF, Arizón JM, González Vilchez F, et al. Primary graft failure after heart transplantation: characteristics in a contemporary cohort and performance of the RADIAL risk score. J Heart Lung Transplant. 2013;32:1187-95.

4. Gohra H, McDonald TO, Verrier ED, Aziz S. Endothelial loss and regeneration in a model of transplant arteriosclerosis. Transplantation. 1995;60:96-102.

5. Russell PS, Chase CM, Winn HJ, Colvin RB. Coronary atherosclerosis in transplanted mouse hearts. I. Time course and immunogenetic and immunopathological considerations. Am J Pathol. 1994;144:260-74.

6. Fedak PWM, Rao V, Verma S, Ramzy D, Tumiati L, Miriuka S, et al. Combined endothelial and myocardial protection by endothelin antagonism enhances transplant allograft preservation. J Thorac Cardiovasc Surg. 2005; 129:407-15.

7. Ramzy D, Rao V, Mallidi H, Tumiati LC, Xu N, Miriuka S, et al. Cardiac allograft preservation using donor-shed blood supplemented with L-arginine. J Heart Lung Transplant. 2005;24:1665-72.

8. Rao V, Feindel CM, Weisel RD, Boylen P, Cohen G. Donor blood perfusion improves myocardial recovery after heart transplantation. J Heart Lung Transpl. 1997; 16:667-73.

9. Wicomb WN, Cooper DK, Barnard CN. Twenty-four-hour preservation of the pig heart by a portable hypothermic perfusion system. Transplantation. 1982; 34:246-50.

10. Junger WG, Coimbra R, Liu FC, Herdon-Remelius C, Junger W, Junger H, et al. Hypertonic saline resuscitation: a tool to modulate immune function in trauma patients? Shock. 1997;8:235-41.

11. Murao Y, Hoyt DB, Loomis W, Namiki S, Patel N, Wolf P, et al. Does the timing of hypertonic saline resuscitation affect its potential to prevent lung damage? Shock. 2000;14:18-23.

12. Shields CJ, Winter DC, Manning BJ, Wang JH, Kirwan WO, Redmond HP. Hypertonic saline infusion for pulmonary injury due to ischemia-reperfusion. Arch Surg. 2003;138:9-14.

13. Vollmar B, Lang G, Menger MD, Messmer K. Hypertonic hydroxyethyl starch restores hepatic microvascular perfusion in hemorrhagic shock. Am J Physiol. 1994;266(5 Pt 2):H1927-34.

14. Waagstein LM, Wennberg E, Waagstein F, Haljamäe H. Hypertonic saline without or with dextran-70 in the treatment of experimental acute myocardial ischemia and reperfusion. Crit Care Med. 1999;27:605-16.

15. Badiwala MV, Ramzy D, Tumiati LC, Tepperman ED, Sheshgiri R, Prodger JL, et al. Donor pretreatment with hypertonic saline attenuates primary allograft dysfunction: a pilot study in a porcine model. Circulation. 2009; 120(11 Suppl):S206-14.

16. Baan J, van der Velde ET, de Bruin HG, Smeenk GJ, Koops J, van Dijk AD, et al. Continuous measurement of left ventricular volume in animals and humans by conductance catheter. Circulation. 1984;70:812-23.

17. Szwarc RS, Mickleborough LL, Mizuno S, Wilson GJ, Liu P, Mohamed S. Conductance catheter measurements of left ventricular volume in the intact dog: parallel conductance is independent of left ventricular size. Cardiovasc Res. 1994;28:252-8.

18. Pober JS, Orosz CG, Rose ML, Savage CO. Can graft endothelial cells initiate a host anti-graft immune response? Transplantation. 1996;61:343-9.

19. Poston RS Jr, Billingham ME, Pollard J, Hoyt EG, Robbins RC. Effects of increased ICAM-1 on reperfusion injury and chronic graft vascular disease. Ann Thorac Surg. 1997;64:1004-12.

20. Ginis I, Mentzer SJ, Li X, Faller DV. Characterization of a hypoxia-responsive adhesion molecule for leukocytes on human endothelial cells. J Immunol. 1995; 155:802-10.

21. Lefer AM, Lefer DJ. The role of nitric oxide and cell adhesion molecules on the microcirculation in ischaemia-reperfusion. Cardiovasc Res. 1996;32: $743-51$. 
22. Tanaka H, Swanson SJ, Sukhova G, Schoen FJ, Libby P. Smooth muscle cells of the coronary arterial tunica media express tumor necrosis factor-alpha and proliferate during acute rejection of rabbit cardiac allografts. Am J Pathol. 1995; 147:617-26.

23. Sidi A, Muehlschlegel JD, Kirby DS, Kirby RR, Lobato EB. Treating ischemic left ventricular dysfunction with hypertonic saline administered after coronary occlusion in pigs. J Cardiothorac Vasc Anesth. 2007;21: 400-5.

24. Fischer M, Dahmen A, Standop J, Hagendorff A, Hoeft A, Krep H. Effects of hypertonic saline on myocardial blood flow in a porcine model of prolonged cardiac arrest. Resuscitation. 2002;54:269-80.

25. Deutsch MA, Kaczmarek I. Letter by Deutsch and Kaczmarek regarding article, "Donor pretreatment with hypertonic saline attenuates primary allograft dysfunction: a pilot study in a porcine model" Circulation. 2010;121:e393. author reply: e394.

26. Harada K, Franklin A, Johnson RG, Grossman W, Morgan JP. Acidemia and hypernatremia enhance postischemic recovery of excitation-contraction coupling. Circ Res. 1994;74:1197-209.

27. Sanada S, Komuro I, Kitakaze M. Pathophysiology of myocardial reperfusion injury: preconditioning, postconditioning, and translational aspects of protective measures. Am J Physiol Heart Circ Physiol. 2011;301:H1723-41.

28. Zakaria el R, Tsakadze NL, Garrison RN. Hypertonic saline resuscitation improves intestinal microcirculation in a rat model of hemorrhagic shock. Surgery. 2006;140:579-87; discussion: 587-8.
29. Verma S, Maitland A, Weisel RD, Li SH, Fedak PW, Pomroy NC, et al. Hyperglycemia exaggerates ischemia-reperfusion-induced cardiomyocyte injury: reversal with endothelin antagonism. J Thorac Cardiovasc Surg. 2002; 123:1120-4.

30. Verma S, Maitland A, Weisel RD, Fedak PW, Li SH, Mickle DA, et al. Increased endothelin-1 production in diabetic patients after cardioplegic arrest and reperfusion impairs coronary vascular reactivity: reversal by means of endothelin antagonism. J Thorac Cardiovasc Surg. 2002;123:1114-9.

31. Mazhar R, Samenesco A, Royston D, Rees A. Cardiopulmonary effects of 7.2\% saline solution compared with gelatin infusion in the early postoperative period after coronary artery bypass grafting. J Thorac Cardiovasc Surg. 1998;115 178-89.

32. Dehoux M, Philip I, Chollet-Martin S, Boutten A, Hvass U, Desmonts JM, et al Early production of interleukin-10 during normothermic cardiopulmonary bypass. J Thorac Cardiovasc Surg. 1995;110:286-7.

33. Ma XL, Weyrich AS, Lefer DJ, Lefer AM. Diminished basal nitric oxide release after myocardial ischemia and reperfusion promotes neutrophil adherence to coronary endothelium. Circ Res. 1993;72:403-12.

34. Hausenloy DJ, Yellon DM. Ischaemic conditioning and reperfusion injury Nat Rev Cardiol. 2016;13:193-209.

Key Words: heart transplantation, myocardial protection, myocardial ischemia/reperfusion injury 


\section{SUPPLEMENTARY MATERIAL. METHODS Donor Procedure}

Donor procedures were performed as described previously. ${ }^{15}$ In brief, the animals were anesthetized with intramuscular ketamine $(30 \mathrm{mg} / \mathrm{kg})$ and inhalational isofluorane $(1 \%-5 \%)$. Following median sternotomy, umbilical tapes were placed around the superior and inferior vena cava to permit adjustment of cardiac preload by caval snaring. A small apical ventriculotomy was created to permit insertion of an intraventricular Millar pressure catheter and a conductance catheter for continuous measurements of the LV pressure-volume relationship. Small myocardial samples from the ventriculotomy site were snap-frozen for further analysis. Pulmonary vascular resistance was assessed using a Swan-Ganz catheter.

After baseline hemodynamic and metabolic measurements were obtained, arterial and coronary sinus blood samples were drawn just before aortic crossclamping. After crossclamping, $1 \mathrm{~L}$ of Celsior solution was infused into the aortic root at $4{ }^{\circ} \mathrm{C}$ to achieve cardioplegic arrest, closely mimicking our current clinical practice. Following arrest, the donor heart was extracted, placed in a jar containing $1 \mathrm{~L}$ of hypothermic cardioplegic solution, and stored on ice.

\section{Recipient Procedure}

Initiation of the recipient procedure was adjusted according to the time of donor organ procurement for a total ischemic interval of 8 hours, as described previously. ${ }^{15}$ Previous studies from our laboratory have demonstrated that $<20 \%$ of hearts will successfully maintain adequate hemodynamics following even shorter periods of ischemia. ${ }^{6-8}$ After the recipient aorta was crossclamped, orthotopic transplantations using a standard atria-to-atria technique were performed. Following transplantation, all hearts were reperfused for 60 minutes. After reperfusion, $1 \mathrm{~g}$ of calcium chloride was given to all animals, and weaning from CPB was attempted. Weaning from CPB was deemed successful if the animal maintained a mean systemic pressure of $60 \mathrm{mmHg}$ for 30 minutes.

Immediately after complete weaning from $\mathrm{CPB}$, hemodynamic parameters were recorded and LV function was assessed as described below. After determination of success or failure to wean from $\mathrm{CPB}$, the hearts were excised for assessment of coronary endothelial function.

\section{Outcome Analysis}

Endothelial-dependent and endothelial-independent vascular relaxation were assessed in vitro by constructing concentration-response curves with a small-vessel myograph for isometric tension recording as described previously. ${ }^{15}$ In brief, isolated segments of the LAD coronary artery were cleaned of fat and connective tissue following transplantation. Control assessments were performed on coronary segments obtained from the recipient's native heart.

The tissue rings were exposed to phenylephrine to elicit maximal vasoconstriction. Endothelium-dependent vasoreactivity was assessed based on a graded vasodilatory response to bradykinin $(0.25-1.0 \mathrm{nmol} / \mathrm{L})$, and similarly, endothelium-independent vasoreactivity was assessed based on the dose-response to sodium nitroprusside ( $10 \mathrm{nmol} / \mathrm{L}$ to $5 \mu \mathrm{mol} / \mathrm{L}$ ). The percentage of maximum relaxation from preconstruction was compared between groups. The sensitivity to vasospasm was also assessed by exposing vessel segments to graded concentrations of ET-1 (0.05$15 \mathrm{nmol} / \mathrm{L}$ ) to yield $\mathrm{Cmax} \%$, calculated as maximum tension increase from baseline. Data were collected with AcqKnowledge software (BIOPAC Systems, Goleta, Calif).

\section{Biochemical Analysis}

Myocardial metabolism was assessed by obtaining simultaneous arterial and coronary sinus blood samples and full-thickness LV myocardial biopsy specimens. Blood samples were obtained at baseline, before donor organ arrest, and at 30-minute intervals during organ storage. In addition, blood samples were obtained during each cardioplegic dose of the recipient operation and at 15-minute intervals during the reperfusion period. Myocardial biopsy specimens were assessed for 8-isoprostane (a marker of lipid peroxidation), myeloperoxidase activity, TNF- $\alpha$ (a marker of cell-mediated endothelial injury), ET-1, and eNOS activity. Lung biopsy specimens were taken from the recipient animals before HTS infusion (baseline control) and at 5 and 60 minutes after transplantation.

The serum concentrations of sodium and lactate were determined by analysis of arterial and coronary sinus whole blood samples using an iSTAT point-of-care whole blood analyzer (Abbott Laboratories, Abbott Park, Ill). Coronary sinus blood plasma TNF- $\alpha$ levels were measured using a porcine-specific enzyme-linked immunosorbent assay kit (R\&D Systems, Minneapolis, Minn), and myocardial 8-isoprostane levels were assayed with an enzyme-linked immunosorbent assay kit (Cayman Chemical Company, Ann Arbor, Mich), following the manufacturer's directions. Lung tissue TNF- $\alpha$, IL-2, IL-6, and IL-10 were detected by standard Western blot techniques using porcine-specific monoclonal antibodies (R\&D Systems). Beta-actin was detected as a loading control for all blots. X-ray films were analyzed using a Bio-Rad GS-800 calibrated densitometer and Bio-Rad Quantity One software (Bio-Rad, Hercules, Calif). 